\title{
Proservação de tratamentos endodônticos realizados na clínica odontológica
}

\author{
Follow-up of endodontic treatments performed in the dental clinic
}

\section{Proservación de tratamientos de endodoncia realizados en la clínica dental}

\section{Resumo}

O objetivo deste estudo foi realizar a proservação dos tratamentos endodônticos, através da observação clínica e radiográfica, o tipo de tratamento foi aplicado, identificar os grupos dentários que foram tratados, verificar quantos casos tiveram sucesso, levantar em quantas sessões foi realizado o tratamento endodôntico, levantar o tipo de medicação intracanal foi utilizada, levantar quais soluções irrigadoras e técnicas de instrumentação foram utilizadas, qualidade das radiografias, qualidade da obturação e levantar quantos dentes tratados endodonticamente foram restaurados e quantos dentes foram perdidos. Foram selecionados 100 prontuários de pacientes que realizaram tratamento endodôntico na clínica odontológica. Foi possível realizar a proservação clínica e radiográfica de 44 dentes, dos quais 30 foram incisivos e 14 pré-molares. As radiografias foram avaliadas por duas endodontistas calibradas. Os resultados mostraram que todos dentes foram classificados como tendo qualidade de tratamento satisfatória. Quando ao limite de obturação, não foi encontrado nenhum dente sobreobturado ou subobturado, todos $(n=100)$ estavam obturados no limite cemento-dentina-canal (CDC). Com relação à condensação do material 
obturador, todos os dentes (100\%) apresentaram condensação adequada, observada pela radiopacidade da obturação na radiografia. Em relação ao tipo de tratamento mais aplicado foi a necropulpectomia, em 96 dentes com lesão $(78,57 \%)$ e 4 dentes sem lesão (14,28\%). Foi realizado apenas 1 biopulpectomia $(7,15 \%)$. Com relação a medicação intracanal, o hidróxido de cálcio foi utilizado em quase a totalidade dos casos, calen com PMCC $(35,71 \%)$ e calen $(28,57 \%)$ seguido por formocresol $(21,42)$ e otosporin $(7,15 \%)$, o hipoclorito de sódio foi a substância usado em todos os casos $(100 \%)$. Com relação a função mastigatória, $80(85,71 \%)$ estavam restaurados com resina composta, 10 fazia parte de uma ponte fixa $(7,15 \%)$ e 10 estavam fraturadas $(7,15 \%)$. Pode-se concluir que a maioria dos dentes que foram avaliados clínico e radiograficamente observou-se o êxito do tratamento endodôntico, inclusive com regressão de lesão periapical.

Palavras-chave: Proservação; Qualidade do tratamento endodôntico; Odontologia.

\begin{abstract}
The objective of this study was to perform endodontic follow-up, through clinical and radiographic observation, or the type of treatment used, to identify groups of teeth that were used, to verify the cases of inappropriate use, to use the first attempts of endodontic treatment, to raise the type of intracanal medication that was used, survey which solutions and techniques of irrigated instrumentation used, quality of radiographs, quality of filling and use of endodontically used teeth were restored and which were lost. One hundred medical records of patients who underwent endodontic treatment at the dental clinic were selected. It was possible to perform a clinical and radiographic proservation of 44 teeth, of which 30 were incisors and 14 premolars. The radiographs were evaluated by two calibrated endodontists. The results shown for all teeth were classified as having satisfactory treatment quality. Regarding the filling limit, no overfilled or underfilled teeth were found, all $(n=100)$ were filled at the limit of the cementum dental canal (CDC). Regarding the condensation of the material obtained, all teeth $(100 \%)$ were adequately condensed, observed by the radiopacity of the obtained on the radiography. Regarding the type of treatment most applied to necropulpectomy, in 96 teeth with injury (78.57\%) and 4 teeth without injury (14.28\%). Only 1 biopulpectomy (7.15\%) was performed. Regarding intracanal medication, calcium hydroxide was used in almost all cases, caliber with PMCC (35.71\%) and caliber $(28.57 \%)$ followed by formocresol $(21.42)$ and otosporin $(7.15 \%)$, sodium hypochlorite was used in all cases $(100 \%)$. Regarding masticatory function, $80(85.71 \%)$ were restored with composite resin, 10 with part of a fixed bridge $(7.15 \%)$ and 10 were fractured $(7.15 \%)$. It can be concluded that most teeth are clinically and radiographically involved in endodontic treatment, including periapical regression.
\end{abstract}

Keywords: Proservation; Quality of endodontic treatment; Dentistry.

\title{
Resumen
}

El objetivo de este estudio fue realizar la continuación de los tratamientos de endodoncia, a través de la observación clínica y radiográfica, el tipo de tratamiento que se aplicó, identificar los grupos dentales que fueron tratados, verificar cuántos casos tuvieron éxito, relevar en cuántas sesiones la endodoncia Se realizó el tratamiento, se examinó el tipo de medicación intracanal utilizada, se examinó qué soluciones de irrigación y técnicas de instrumentación se utilizaron, la calidad de las radiografías, la calidad de los empastes y se examinó cuántos dientes tratados endodónticamente se restauraron y cuántos dientes se perdieron. Se seleccionaron cien historias clínicas de pacientes que se sometieron a tratamiento de endodoncia en la clínica dental. Se pudo realizar el seguimiento clínico y radiográfico de 44 dientes, de los cuales 30 eran incisivos y 14 premolares. Las radiografías fueron evaluadas por dos endodoncistas calibrados. Los resultados mostraron que todos los dientes se clasificaron como de calidad de tratamiento satisfactoria. En cuanto al límite de obturación, no se encontraron dientes sobrellenados o subllenados, todos $(\mathrm{n}=100)$ fueron obturados en el límite cemento-canal dentinario (CDC). En cuanto a la condensación del material de obturación, todos los dientes $(100 \%)$ presentaron una adecuada condensación, observada por la radiopacidad de la obturación en la radiografía. El tipo de tratamiento más aplicadas fue la necropulpectomía, en 96 dientes con lesiones (78,57\%) y 4 dientes sin lesiones $(14,28 \%)$. Solo se realizó 1 biopulpectomía $(7,15 \%)$. En cuanto a la medicación intracanal, se utilizó hidróxido de calcio en casi todos los casos, calen con PMCC $(35,71 \%)$ y calen $(28,57 \%)$ seguido de formocresol $(21,42)$ y otosporina $(7,15 \%)$, siendo hipoclorito de sodio la sustancia utilizada en todos los casos $(100 \%)$. En cuanto a la función masticatoria, $80(85,71 \%)$ fueron restauradas con resina compuesta, 10 formaron parte de un puente fijo $(7,15 \%)$ y 10 se fracturaron $(7,15 \%)$. Se puede concluir que la mayoría de los dientes que fueron evaluados clínica y radiográficamente mostraron un tratamiento endodóntico exitoso, incluida la regresión de la lesión periapical.

Palabras clave: Proservación; Calidad del tratamiento de endodoncia; Odontología.

\section{Introdução}

O tratamento endodôntico tem como objetivo a promoção de um ambiente adequado para que o organismo possa obter o reparo dos tecidos periapicais após a intervenção terapêutica e, com isso, o dente poder retornar as suas funções normais. Para o alcance de tal objetivo, é necessário que se estabeleça a limpeza e modelagem com objetivo de desinfetar os 
sistemas de canais radiculares, a obturação e o selamento coronário (Ferreira et al., 2007; Ferreira et al., 2013; Barbosa-Ribeiro et al., 2021).

A endodontia está relacionada com o estudo da forma, função, saúde, agressões e enfermidades da polpa dentária e região periapical, bem como suas prevenções e tratamentos. As etapas de uma Endodontia com qualidade envolvem desde a anamnese à restauração definitiva e proservação (Gaspar-Júnior et al., 2009; Patriota et al., 2020; Alves-Silva et al., 2020). Entretanto, no meio desses extremos vale ressaltar que a preparação do acesso à cavidade, determinação da profundidade operacional ou comprimento de trabalho e a obturação do sistema de canais radiculares são fases de extrema perfeição. A atenção em todas as etapas eleva a qualidade dos tratamentos endodônticos que pode ser verificada através de uma radiografia que mostre o ápice da raiz claramente identificável e uma obturação endodôntica com o trajeto do canal radicular original totalmente preenchido, devendo afilar-se em direção ao ápice e nenhum espaço entre a massa obturadora e as paredes do canal devem ser visualizadas (Alves-Silva et al., 2020).

Estudos correlacionam o sucesso e o fracasso dos tratamentos endodônticos à qualidade da obturação, seu limite apical, presença prévia e extensão da lesão periapical, infiltração apical e coronária, complexidade anatômica e presença da lesão cárie após o tratamento (Gaspar-Júnior et al., 2009; Alves-Silva et al., 2020).

Para avaliar o sucesso de um tratamento endodôntico é necessário realizarmos um controle clínico e radiográfico do paciente, onde são avaliados os seguintes critérios: dor, odor, edema, fístula, presença ou não de lesão periapical. Se todas as etapas do tratamento endodôntico forem realizadas adequadamente, espera-se que, após o período de 1 a 2 anos de avaliação, o sucesso seja alcançado (Giusti et al., 2007; Patriota et al., 2020). Além disso, é importante diagnosticarmos se o elemento dentário que recebeu o tratamento endodôntico foi restaurado adequadamente, devolvendo ao paciente a função mastigatória (Gonçalvez, 2007).

Muitos estudos de avaliação radiográfica dos resultados obtidos com os tratamentos endodônticos, analisaram a obturação do sistema de canais radiculares, relacionando à qualidade das obturações no quesito de condensação homogeneidade o limite apical. O sucesso dos tratamentos endodônticos atualmente margeia-se em altos índices de porcentagem. Com o aprimoramento das técnicas endodônticas e restauradoras e o desenvolvimento dos materiais tem-se observado uma melhora significativa na qualidade dos resultados dos tratamentos endodônticos (Lage-Marques et al., 1996; Pereira et al., 2008; Alves-Silva et al., 2020).

O sucesso do tratamento endodôntico depende, sobretudo, do conhecimento da anatomia pulpar, patologia e microbiologia, além do correto diagnóstico dos casos e uma adequada habilidade para a execução das fases do tratamento por completo (Alves-Silva et al., 2021). A determinação do sucesso e insucesso endodôntico deve basear-se em critérios bem definidos, englobando características clínicas e aspectos radiográficos que sejam condizentes com o processo de reparação tecidual como: ausência de dor e edema, função normal do dente, desaparecimento ou diminuição da lesão periapical (AlvesSilva et al., 2021).

A busca do sucesso dos tratamentos endodônticos deve ser referencial em todos os momentos da Endodontia e, que a mensuração da qualidade precisa ser avaliada sobre vários aspectos conjuntamente: instrumentação em todo o canal radicular, irrigação abundante, obturação tridimensional e restauração definitiva bem realizada (Louzada et al., 2020; ArrudaVasconcelos et al., 2021).

Quando o tratamento endodôntico falha, indica-se, como primeira opção, o retratamento do (s) canal (is) radicular (es) desde que haja condição para tal. Outra opção é realizar a cirurgia apical, com intuito de eliminar o biofilme extra-radicular. E, em alguns casos realizam-se ambos os tratamentos (Trvassos et al., 2005; Imura, 2004; Patriota et al., 2020; Alves-Silva et al., 2021). 
O objetivo desta pesquisa foi realizar a proservação dos tratamentos endodônticos nos dentes tratados na clínica odontológica da Universidade Potiguar, através da observação clínica e radiográfica de tratamentos realizados entre os anos de 2011 e 2015.

\section{Metodologia}

Seguindo os preceitos estabelecidos pela resolução 196/96 do Ministério da Saúde, este estudo foi devidamente registrado na Base de Registro de Pesquisa envolvendo seres humanos (Plataforma Brasil) e submetido à apreciação e aprovação do Comitê de Ética e Pesquisa da Universidade Potiguar-UnP, com registro de número do CAAE 57260816.9.0000.5296.

Trata-se de um estudo observacional, retrospectivo, de carácter descritivo de dentes tratados endodonticamente, de pacientes atendidos na clínica da Universidade Potiguar. Inicialmente foram analisadas 500 fichas clínicas de pacientes que foram submetidos a tratamento endodôntico na clínica odontológica da Universidade Potiguar, entre os anos de 2011 a 2015. Dessas fichas, 400 não de encaixaram nos critérios de inclusão, 274 fichas clínicas estavam incompletas, 57 fichas não se encaixaram na faixa etária, 69 radiografias estavam com padrões inadequados. Um total de 100 prontuários de pacientes foram envolvidos na presente pesquisa.

Os critérios de inclusão foram prontuários de pacientes submetidos a tratamento endodôntico, tanto de dentes com polpa vital e necrótica, devidamente preenchidos e contendo radiografias iniciais e finais do tratamento endodôntico de boa qualidade. Foram excluídos da pesquisa: prontuários incompletos, faltando radiografias iniciais ou finais ou com radiografias ilegíveis.

Após seleção dos prontuários, os pacientes foram convidados a realizar uma avaliação clínico-radiográfica, os quais assinaram o Termo de Consentimento Livre e Esclarecido (TCLE).

Os dentes foram radiografados com filmes radiográficos Insight n ${ }^{\circ} .2$ (Kodak, Rochester, EUA) e aparelho de raios X de 10 mA, e 70 KvP (Dabi-Atlante, Ribeirão Preto, SP, Brasil) com exposição de 0,4 segundos, utilizando-se posicionadores radiográficos. O processamento radiográfico foi realizado em câmara escura portátil. Após a secagem, as radiografias foram montadas em cartelas e devidamente identificadas, para posterior avaliação. Para dentes com mais de um canal ou cujos canais radiculares estivessem sobrepostos foi utilizada a técnica de dissociação radiográfica de Clark, para possibilitar a sua visualização. Foi realizada uma análise estatística descritiva dos dados obtidos.

\section{Resultados}

Foram avaliados percentuais dos tipos de tratamentos realizados, sejam, dentes com necrose pulpar (necropulpectomia) com percentual de 78,57\% dos tratamentos realizados, necrose pulpar com lesão periapical com 14,28\% e em casos de biopulpectomias apresentaram 7,14\% dos tratamentos, como mostra no Gráfico 1. 
Gráfico 1 - Tipo de tratamento realizado.

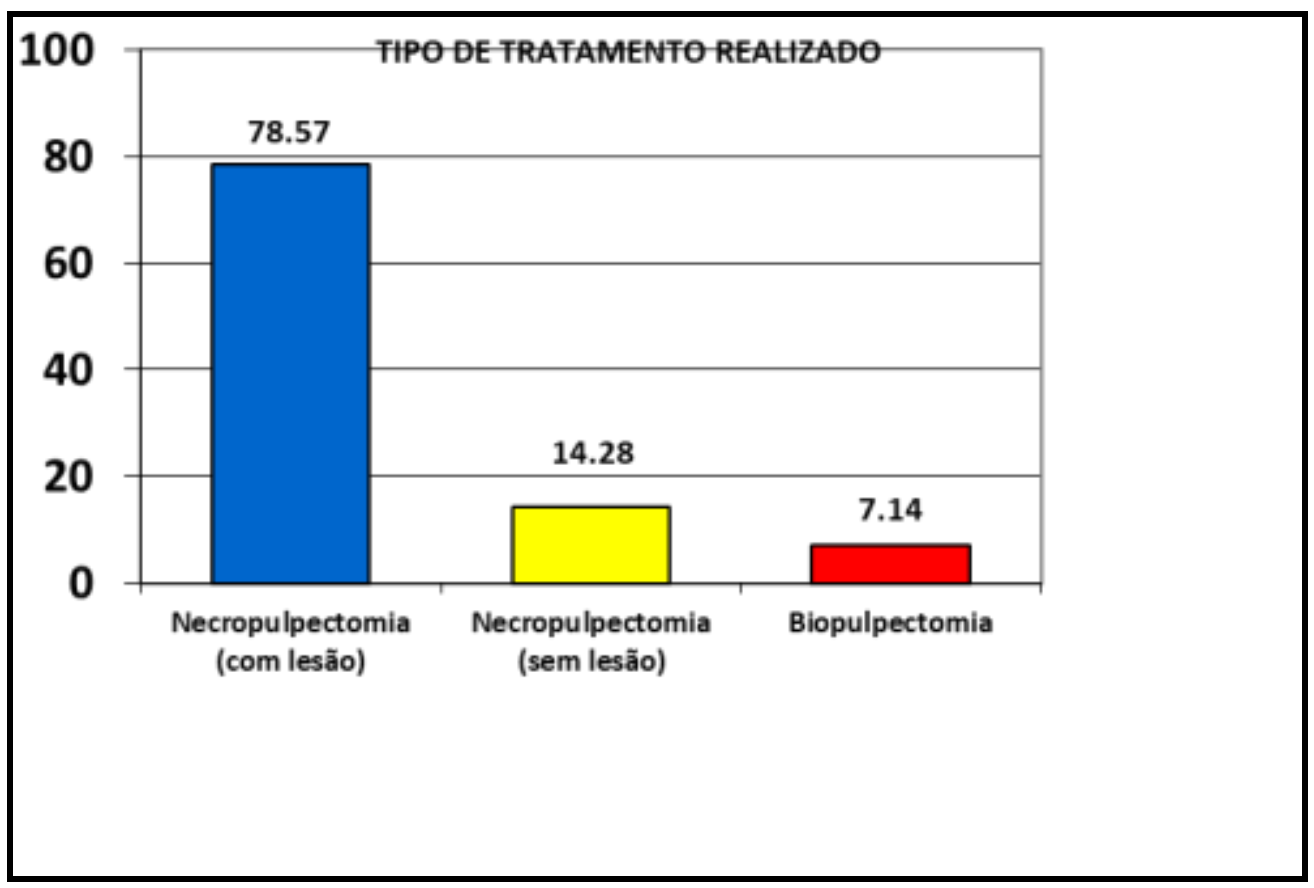

Fonte: Autores.

Em relação a quantidades de sessões realizadas em casa paciente, foi observado que $85,71 \%$ dos casos foram realizados em duas sessões e 14,29\%, foram realizadas em sessão única, visto no Gráfico 2.

Gráfico 2 - Quantidade de sessões realizadas.

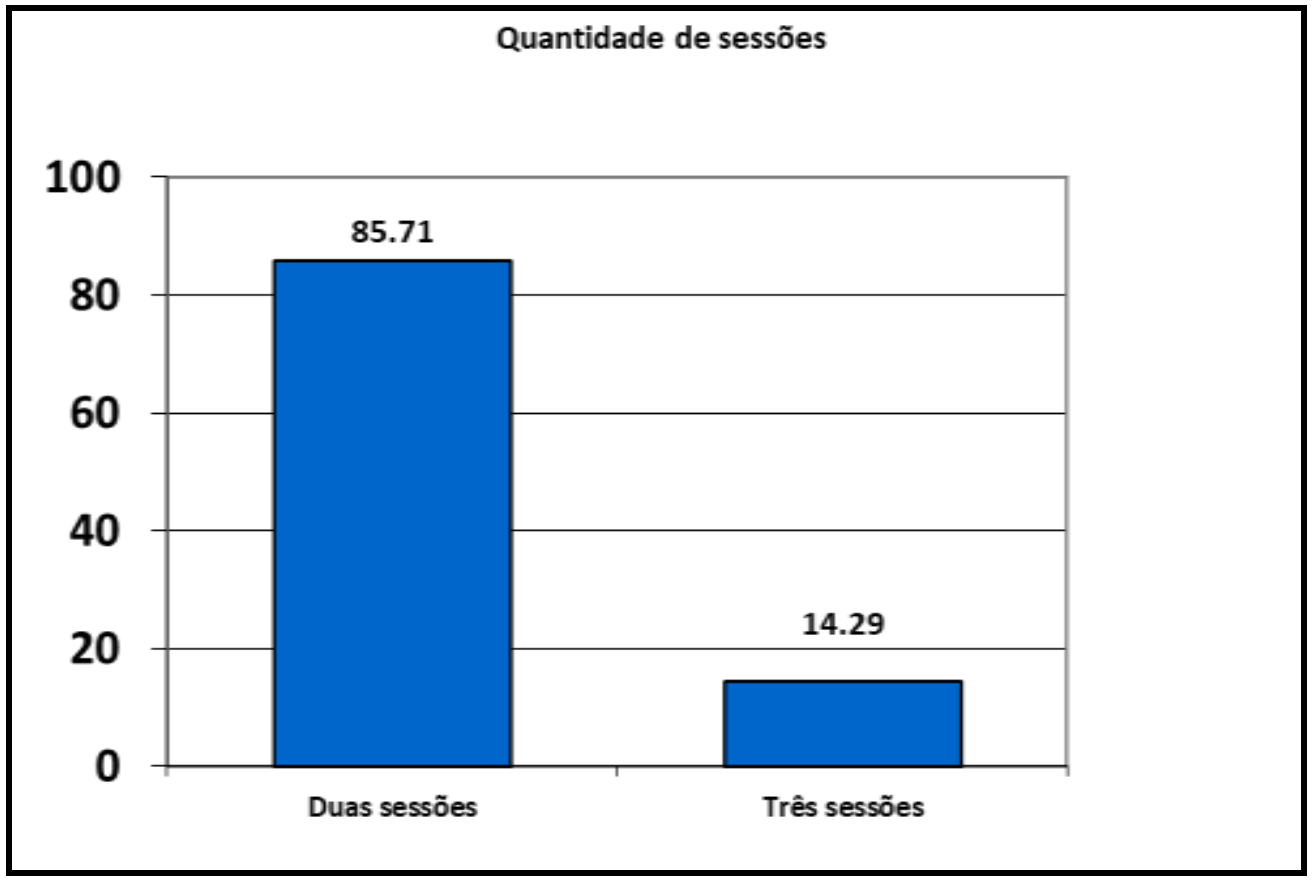

Fonte: Autores.

Foram avaliados tipos de medição intracanal utilizada nos tratamentos endodônticos, onde 35,71\% dos casos foram utlizados calen com PMCC, 28,57\% usaram calen, 21,42\% formocresol, 7,15\% otosporin e 7,15\% dos casos não utlizaram nenhum tipo de medicação. 
Gráfico 3 - Medicações Intracanal canal utilizadas.

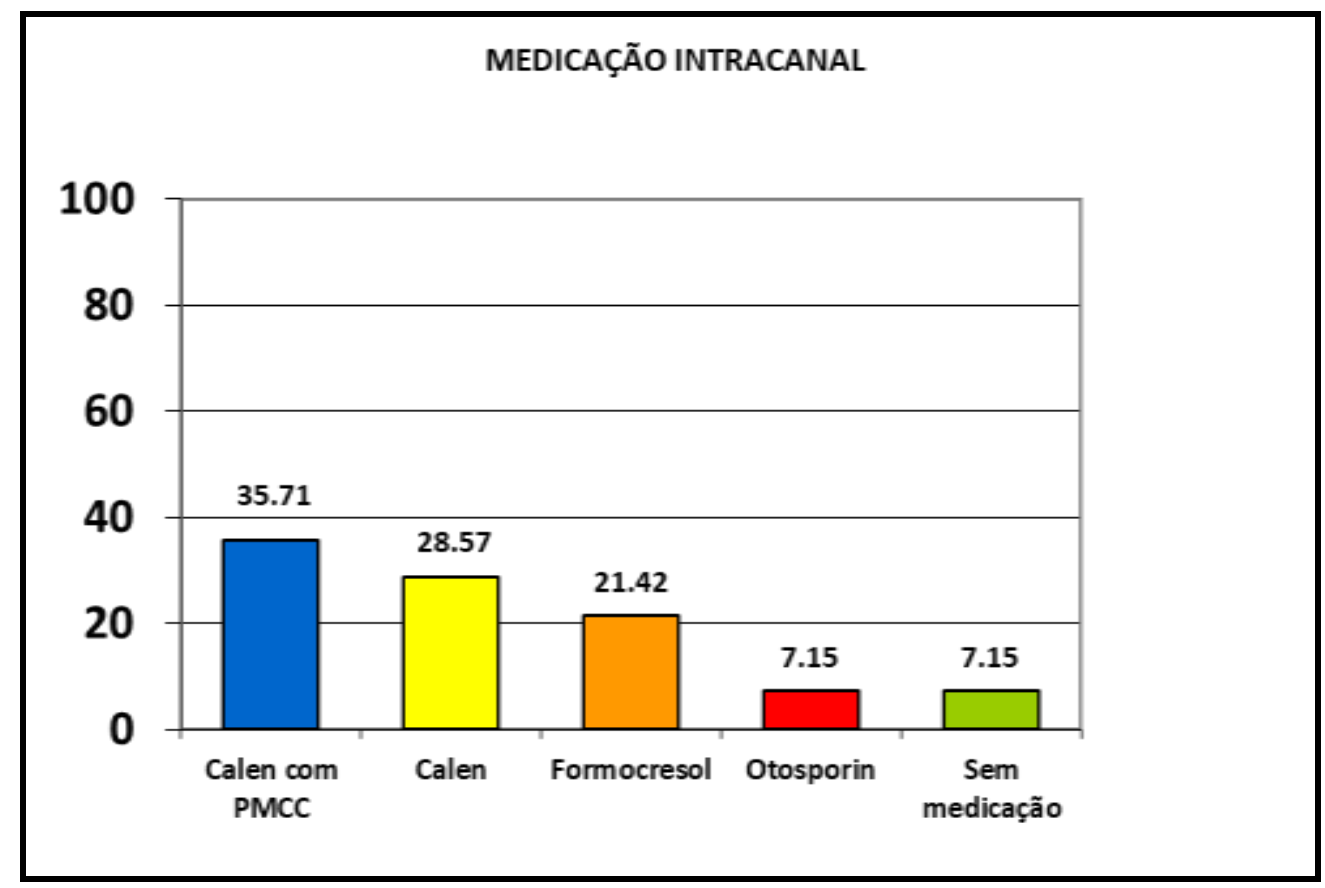

Fonte: Autores.

Em relação ao tipo de solução irrigadora utilizada, foram observados o uso da clorexidina (7,15\%) solução de Milton $(92,85 \%)$ e em $100 \%$ dos tratamentos realizados foi utilizada o EDTA e soro fisiológico na irrigação final, pós preparo biomecânico, como mostra o Gráfico 4.

Gráfico 4 - Tipo de solução irrigadora utilizada no preparo biomecânico.

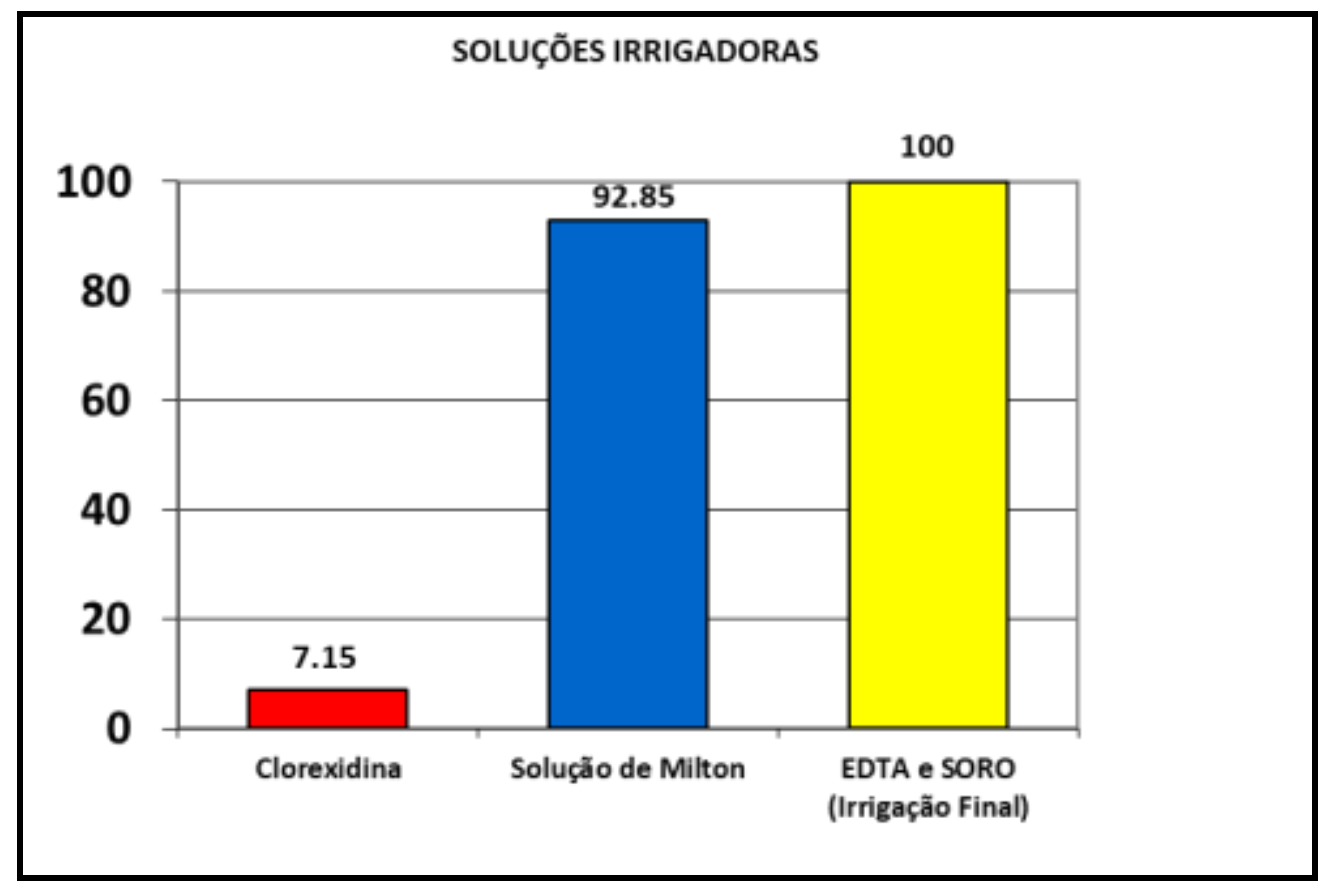

Fonte: Autores. 
Foram realizados tratamento endodôntico em $71,43 \%$ em incisivos e $28,57 \%$ em pré-molares, dados observados no Gráfico 5.

Gráfico 5 - Grupos dentários que receberam tratamento endodôntico.

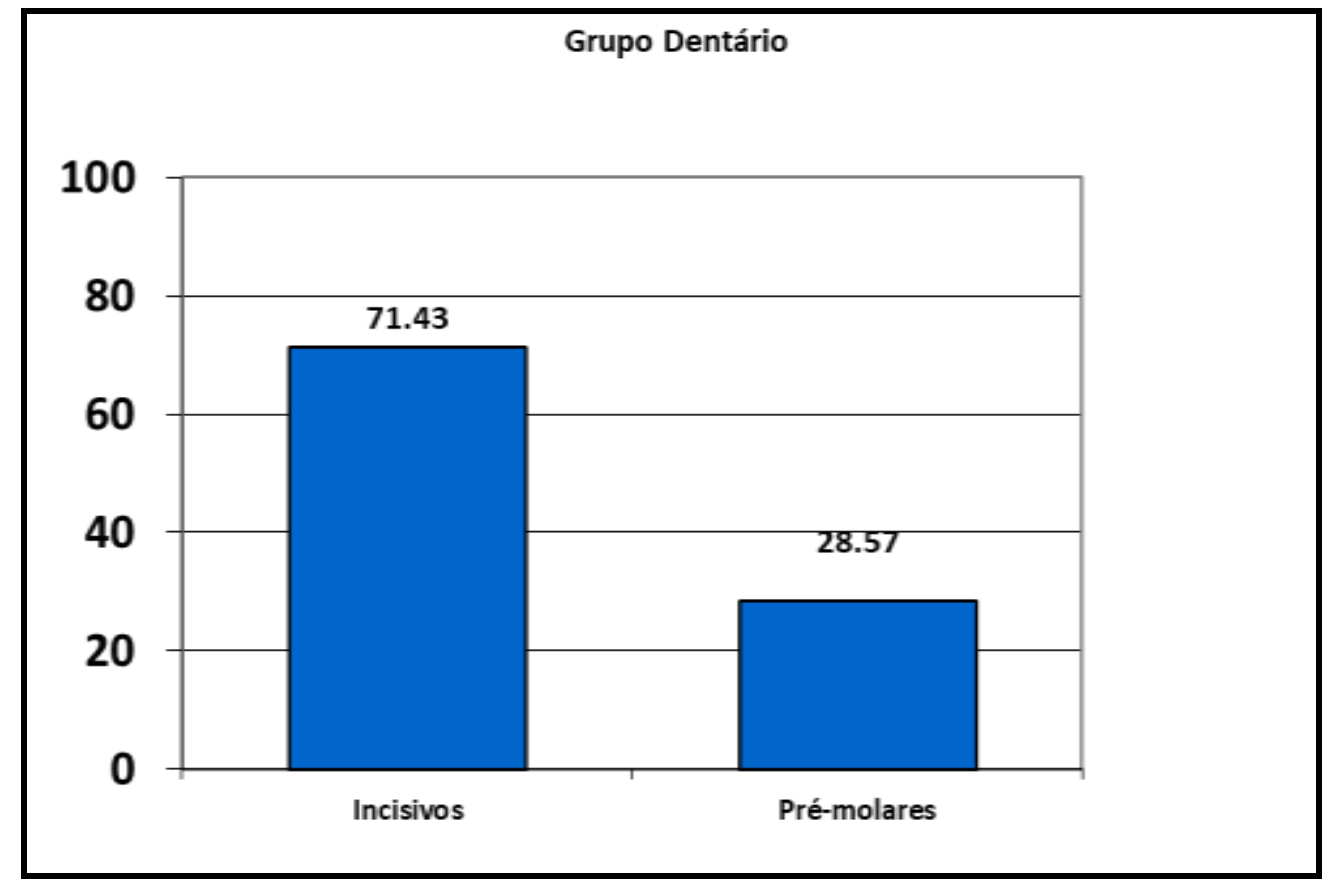

Fonte: Autores.

Em relação a técnica de instrumentação realizados por alunos de graduação, foram observados que 35,71\% dos casos foram realizados utilizando a técnica manual convencional, $50 \%$ dos por meio da técnica escalonada programada e $14,29 \%$ dos casos realizados usando as limas protaper manual, vistas no Gráfico 6.

Gráfico 6 - Técnicas de instrumentação utilizadas.

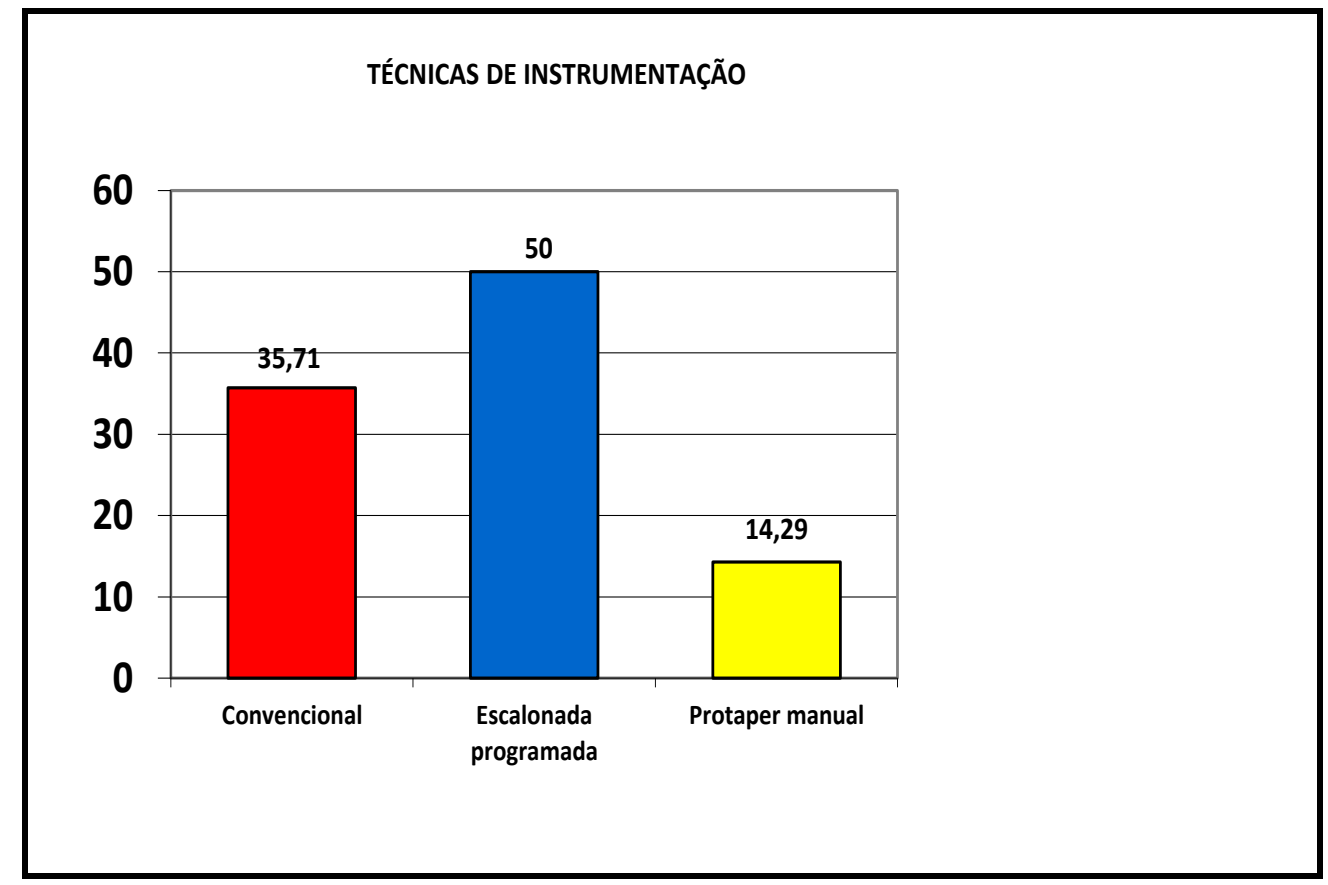

Fonte: Autores. 
O limite apical de obturação também foi avaliado, sendo $100 \%$ dos casos a obturação ter sido realizada no limite cemento-dentina-canal (CDC), como mostra o Gráfico 7.

Gráfico 7 - Limite apical de obturação do canal radicular.

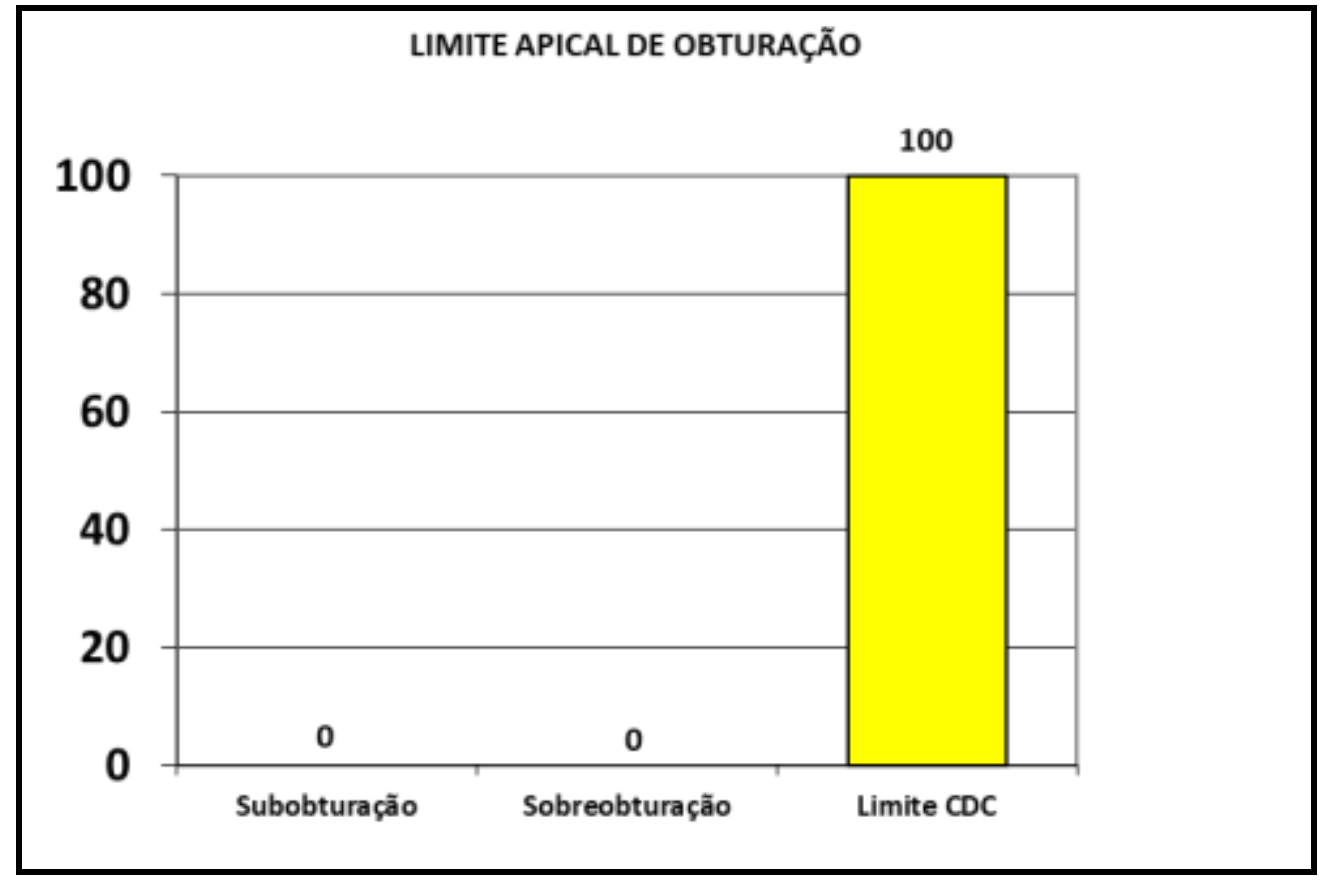

Fonte: Autores.

Em relação a estrutura coronária, $85,71 \%$ dos casos foram restaurados com resina composta, 7,15\% tinha presença de algum tipo de fratura, 7,15\% apresentaram algum tipo de prótese fixa, visto no Gráfico 8.

Gráfico 8 - Estrutura coronária (selamento coronário).

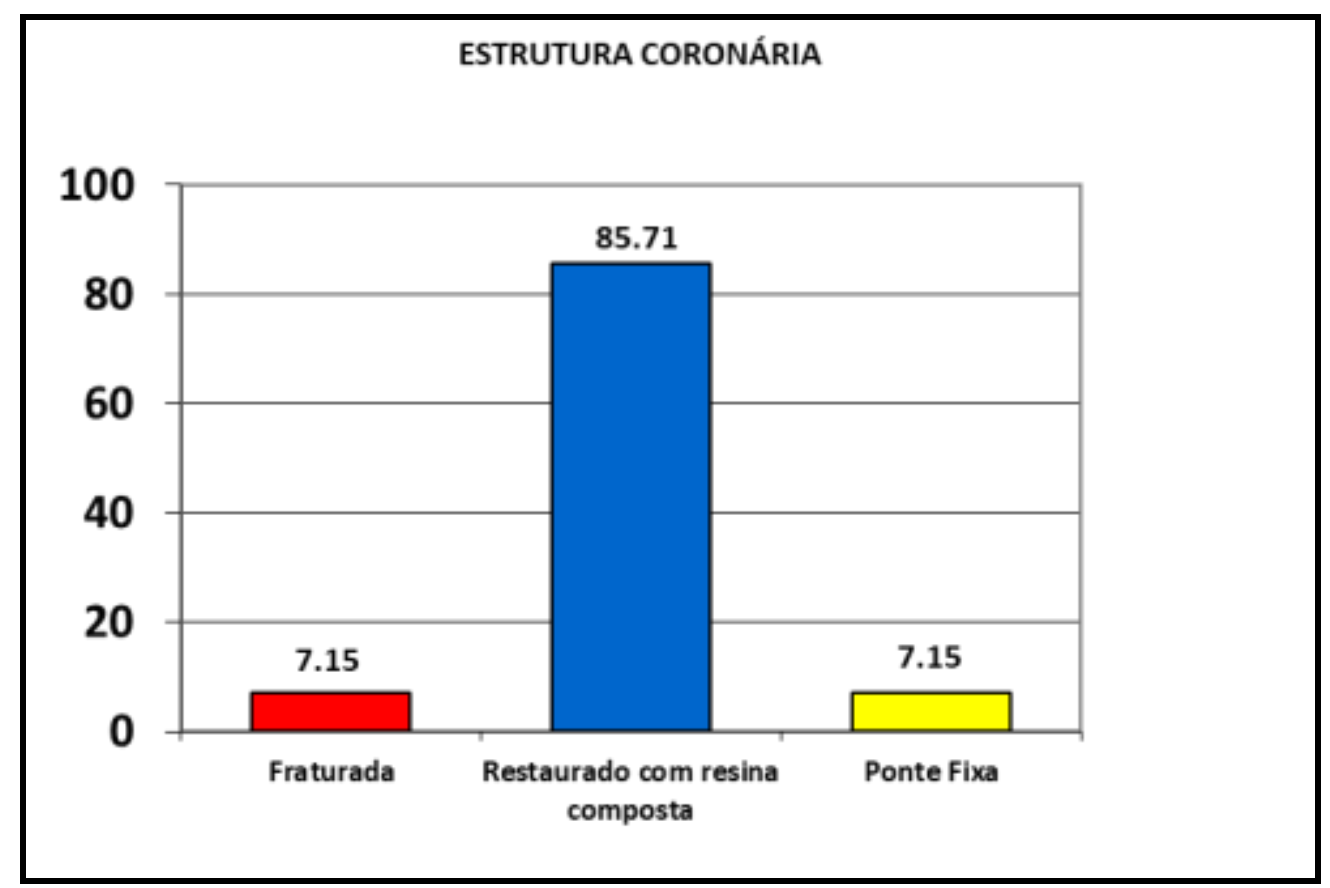

Fonte: Autores. 
Condição periapical e índice de sucesso, $85,71 \%$ dos casos apresentaram radiograficamente sem lesão periapical e 14,29\% com lesão, como mostra o Gráfico 9.

Gráfico 9 - Condição periapical e índice de sucesso do tratamento endodôntico.

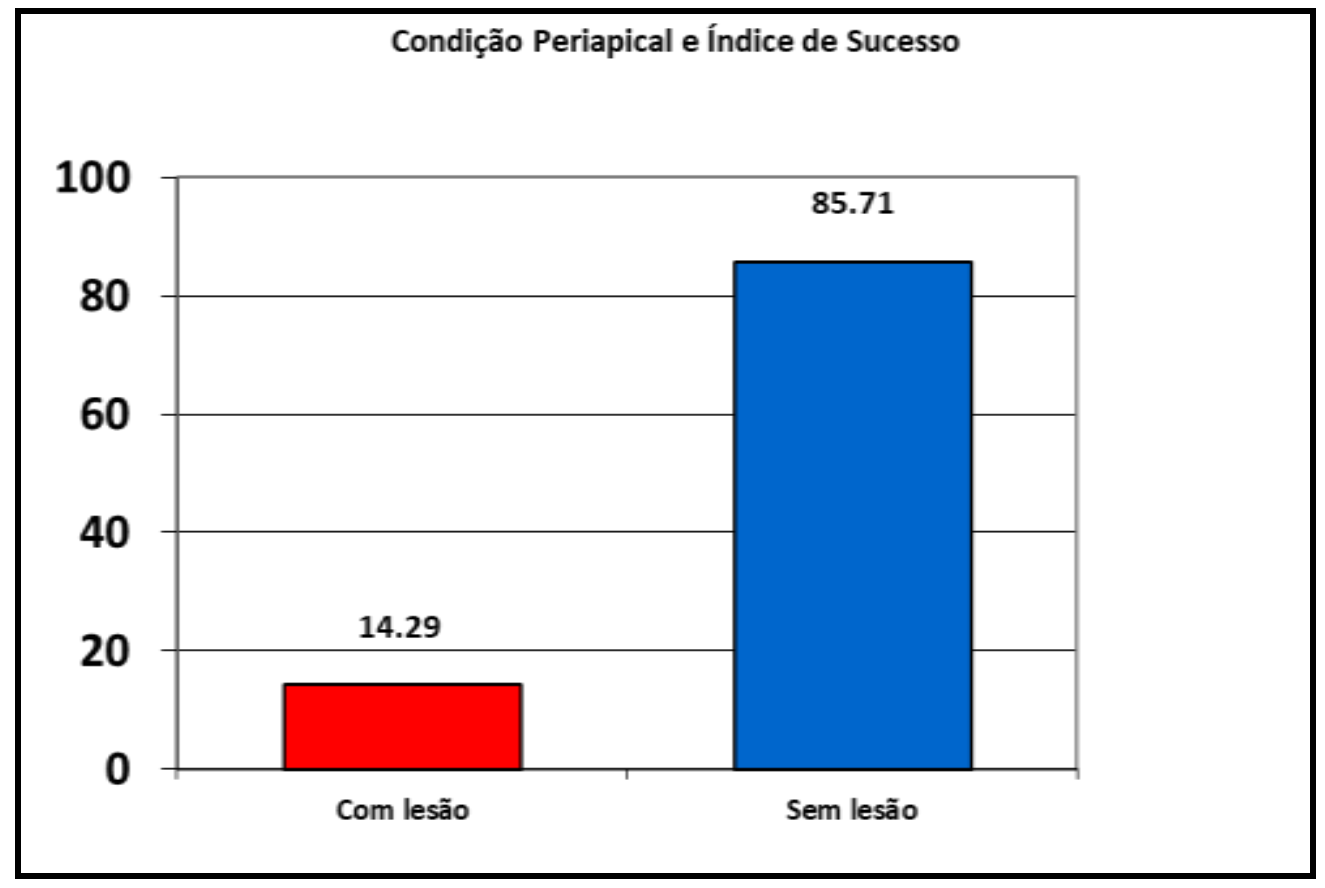

Fonte: Autores.

\section{Discussão}

O sucesso do tratamento endodôntico é demonstrado pela presença da lâmina dura intacta, pelo espaço normal do ligamento periodontal e pela normalidade do osso periapical. Clinicamente, tal sucesso não deve apresentar sinal ou sintoma, e o dente deve estar em função, apresentando restauração coronária e endodôntica satisfatórias (Alves-Silva et al., 2020).

No período de 02/2016 a 11/2016 foram selecionadas 500 fichas de pacientes que foram submetidos ao tratamento endodôntico na clínica odontológica da Universidade Potiguar, porém 100 pacientes entraram nos critérios de inclusão, e foram envolvidos no presente trabalho, sendo que apenas 41 fizeram a radiografia de proservação.

Neste trabalho enfrentamos muitas dificuldades, além do tamanho da amostra, outra dificuldade foi quanto ao preenchimento correto e completo dos dados dos pacientes nos prontuários, as radiografias (inicial e final do tratamento) que estavam ausentes ou sem condições de análise para a comparação com a radiografia de proservação, da organização do arquivo da universidade (dificultando à procura dos prontuários), da entrada dos pacientes que foram resgatados para fazerem a radiografia de acompanhamento da pesquisa na clínica odontológica da Universidade.

O sucesso do tratamento endodôntico é demonstrado pela presença da lâmina dura intacta, pelo espaço normal do ligamento periodontal e pela normalidade do osso periapical. Clinicamente, tal sucesso não deve apresentar sinal ou sintoma, e o dente deve estar em função, apresentando restauração coronária e endodôntica satisfatórias (Bourreau et al., 2020; AlvesSilva et al., 2020). Outros fatores também estão relacionados a falha do tratamento, como a falta de experiência do operador. Como consequência de falha, é constada a presença de sintomatologia após o tratamento, bem como a persistência de radiolucidez apical (Bourreau et al., 2020; Alves-Silva et al., 2021).

De todos os dentes avaliados, nenhum paciente apresentou sinais e sintomas (dor, odor, edema, fístula). E, tanto as radiografias iniciais quanto as finais apresentaram-se com boa qualidade. 
Em relação ao tipo de tratamento mais aplicado foi a necropulpectomia, em 96 dentes com lesão $(78,57 \%)$ e 4 dentes sem lesão (14,28\%). Foi realizado apenas 21 biopulpectomia (7,15\%). De acordo com Lopes et at., 2004, define-se biopulpectomia como a relação da polpa sã ou inflamada, sendo um procedimento microcirúrgico com uma indicação primordial nos casos de pulpite irreversível, sintomática ou assintomática, ou nos quais houve fracasso do tratamento conservador direto (capeamento direto, capeamento indireto ou pulpotomia) e a necropulpectomia é o tratamento de uma infecção que irá de instalar no sistema de canais radiculares, uma vez que a capacidade de defesa tecidual foi perdida em decorrência da necrose (Aveiro et al., 2020).

Além da importância de se prevenir a introdução de novos microorganismos no interior do sistema de canais radiculares, deve-se eliminar a infecção endodôntica para que o tratamento logre êxito. Em casos de necrose pulpar é indicada a obturação do canal em duas sessões, após a permanência de uma medicação intracanal (hidróxido de cálcio com PMCC) (Lopes et at., 2004; Aveiro et al., 2020). Com relação ao número de sessões para realização do tratamento endodôntico observou-se que 82 dentes foram tratados em duas sessões $(85,71 \%)$ e 18 em três sessões (14,29\%). Em relação à medicação intra canal, 12 dentes tilizou otosporin (7,15\%), 24 dentes formocresol (21,42\%), 21 calen (28,57\%), 32 calen com PMCC $(35,71 \%)$ e 11 dentes sem medicação intra canal $(7,15 \%)$.

O hipoclorito de sódio $(\mathrm{NaOCl})$ é a substancia química auxiliar mais empregada no tratamento endodôntico de dentes com necrose pulpar, em concentração variando entre $0,5 \%$ a 5,25\%. Esta substância possui atividade antibacteriana rápida e pronunciada contra uma gama variada de microorganismos, inclusive bactérias comumente isoladas de infecções endodônticas (Lopes et at., 2004; Louzada et al., 2020). As soluções irrigadoras e as medicações intra canais oferecem condições importantes capazes de alterar a microbiota situada no canal radicular. Contudo, devido à difusão da infecção endodôntica, acredita-se que o preparo biomecânico e as soluções irrigadoras bactericidas ainda não são suficientes para se obter a completa desinfecção do sistema de canais radiculares, por isso alguns autores indicam o tratamento em algumas sessões com a medicação intra canal entre elas (Aveiro et al., 2020; Menezes et al., 2021). Neste estudo, 99 dentes foram irrigados foram irrigados com hipoclorito de sódio a 1\% - solução de Milton (100\%), 1 dente foi irrigado com clorexidina (7,15\%) e, todos os dentes foram irrigados com EDTA e soro $(100,0 \%)$ ao final da instrumentação.

O preparo biomecânico é uma das etapas de maior relevância no controle da infecção endodôntica e mais difíceis do tratamento endodôntico, caso ocorra alguma falha na execução de um dos procedimentos pode acarretar o insucesso de toda a terapia, uma vez que contribuem para a permanência do processo infeccioso (Barbosa-Ribeiro et al., 2021). No presente estudo a técnica mais utilizada foi a escalonada programada com 50 dentes (50\%), a convencional com 30 dentes $(35,71 \%)$ e a proteper manual com 20 dentes $(14,29 \%)$.

Com relação ao grupo dentário tratado, 90 dentes foram incisivos e 10 pré-molares. Estudos correlacionam o sucesso e o fracasso dos tratamentos endodônticos à qualidade da obturação, seu limite apical, presença prévia e extensão da lesão periapical, infiltrações apicais e coronária, complexidade anatômica e presença da lesão cárie após o tratamento (Alves-Silva et al., 2020). Neste levantamento, nenhum dente estava sobre ou sub obturado ou obturado totalmente, todos os 100 (100\%) estavam obturados no limite adequado, ou seja, $1 \mathrm{~mm}$ aquém do comprimento real do dente (CRD).

Há algum tempo já é sabido que o futuro do dente como parte integrante do sistema mastigatório não se restringe a uma boa realização da terapia endodôntica, mas também a realização de uma adequada restauração (Patriota et al., 2020; Alves-Silva et al., 2021). Dos 100 dentes que foram radiografados, apenas 10 não estava em função mastigatória, pois estava fraturado $(7,15 \%), 10$ fazia parte de uma ponte fixa $(7,15 \%)$ e 80 estavam restaurados com resina composta $(85,71 \%)$.

Longos períodos de acompanhamento são necessários para avaliação do sucesso do tratamento (Souza, 2006; Gencoglu et al., 2010; Neves et al., 2020). Dos 100 dentes que foram acompanhados nesta pesquisa, 41 foram realizadas radiografias de proservação, destes, 21 obtiveram sucesso no tratamento de necropulpectomia $(78,57 \%), 6$ que estavam com 
lesão não tiveram sucesso delas com relação a sua regressão na sua totalidade $(14,29 \%)$, sendo que esses 3 dentes são do mesmo paciente, talvez por causa do seu problema periodontal e porque tem um dente sem reabilitação. Já o dente que foi submetido à biopulpectomia, 7 (7,15\%) não houve aparecimento de lesão.

É importante enfatizar que só consideramos que um tratamento endodôntico só obterá sucesso a partir do momento que tiver sido realizado o acompanhamento periódico. Tal acompanhamento deverá ser clínico e radiográfico durante um tempo, tal qual foi realizado na pesquisa (Gonçalvez, 2007; Grecca, 2008; Alves-Silva et al., 2020).

Então diante de todos esses resultados da pesquisa, podemos apontar a importância de fazer a proservação de um tratamento endodôntico, principalmente de pacientes como os da pesquisa. Pois só assim podemos ter a confirmação de que o tratamento obteve êxito e analisarmos a qualidade do nosso trabalho, dando assim ao profissional controle total das condições pregressas e atuais do tratamento.

\section{Considerações Finais}

Diante das dificuldades encontradas durante a realização para o levantamento de dados para essa pesquisa, sentimos que é necessário que haja mais tempo para essa coleta de dados para a obtenção de uma amostra mais representativa do índice de sucesso dos tratamentos endodônticos. Podemos concluir que: a maioria dos dentes que foram avaliados clínico e radiograficamente observou-se o êxito do tratamento endodôntico, inclusive com regressão de lesão periapical. Apenas 6 casos não houve êxito completo, pois, o paciente não tinha reabilitado totalmente os dentes.

\section{Agradecimentos}

Este trabalho foi financiado pela Fundação de Amparo à pesquisa do Estado de São Paulo (FAPESP) (número de financiamento: 2015/23479-5/ 2019/09115-1/ 2021/08372-0), CNPQ (número de financiamento: 308162/2014-5) e Coordenação de Aperfeiçoamento de Pessoal de Nível Superior Código Financiamento 001 da CAPES. Os autores negam quaisquer conflitos de interesse.

\section{Referências}

Alves-Silva, E. G., Medeiros, J. B. G., Medeiros, W. D., Dametto, F. R., Arruda-Vasconcelos R, Louzada, L. M., Gomes, B. P. F. A. \& Gadê-Neto, C. R. (2021). Ultrasonic inserts in channel cleaning with fiber posts: in vitro study. Research, Society and Development. $10(2)$. e3481029536.

Alves-Silva, E. G., Souza, P. X., Solano, N. T., Carvalho, R. A., Dametto, F. R., Gomes, B. P. F. A \& Carvalho, L. K. C. G. (2020). Análise da qualidade das radiografias executadas durante os tratamentos endodônticos realizados na graduação da Universidade Potiguar. Archives of health investigation. 9 (3):216221.

Arruda-Vasconcelos, R., Louzada, L. M., Feres, M., Tomson, P. L., Cooper, P. R. \& Gomes, B. P. F. A. (2021). Investigation of microbial profile, levels of endotoxin and lipoteichoic acid in teeth with symptomatic irreversible pulpitis: a clinical study. Int Endod J. 54 (1):46-60

Aveiro, E., Chiarelli-Neto, V. M., De-Jesus-Soares, A., Zaia, A. A, Ferraz, C. C. R., Almeida, J. F. A., Marciano, M. A., Feres, M \& Gomes, B. P. F. A. (2020). Efficacy of reciprocating and ultrasonic activation of $6 \%$ sodium hypochlorite in the reduction of microbial content and virulence factors in teeth with primary endodontic infection. Int Endod J. 53 (5):604-618.

Barbosa-Ribeiro, M., Arruda-Vasoncelos, R., Louzada, L. M., Dos-Santos, D. G., Andreote, F. D \& Gomes, B. P. F. A. (2021). Microbiological analysis of endodontically treated teeth with apical periodontitis before and after endodontic retreatment. Clin Oral Investig. 25 (4):2017-2027

Bourrerau, M. L. S., Frozoni, M. R. S., Mota, M. J. B. B., Zaia, A. A, Lima C. O. Prado, M \& Soares AJ. (2020). Evaluation of single visit endodontic treatment and non-surgical retreatment with foraminal enlargment of teeth with apical periodontitis?. Revista Brasileira de Odontologia. 77 (1):1-7.

Ferreira, H. L. J., Paula, M. V. Q \& Guimarães, S. M. R. (2007). Avaliação radiográfica de obturações de canais radiculares. Rev Odonto Ciênc. 22(58):340345 .

Ferreira, M. A. (2013). Avaliação da Qualidade dos Tratamentos Endodônticos em Centros de Especialidades Odontológicas da Grande Natal- RN. Pesq Bras Odontoped Clin Integr. 13(2):155-60.

Gaspar-Júnior, A. A., Pinheiro, J. T., Couto, G. B. L., Soares, R. P. F \& Neves, C. A. F. (2009). Avaliação radiográfica dos tratamentos endodônticos encontrados nos estudantes de odontologia no Estado de Pernambuco. Clín-Científ. 8(1):29-34. 
Research, Society and Development, v. 10, n. 11, e532101119724, 2021

(CC BY 4.0) | ISSN 2525-3409 | DOI: http://dx.doi.org/10.33448/rsd-v10i11.19724

Gencoglu, N., Pekiner F. N, Gumbru, B \& Helvacioglu, D. (2010). Periapical status and quality of root fillings and coronal restorations in an adult Turkish subpopulation. European Journal of Dentistry. 4 (1): 17-22.

Giusti, E. C., Puertas, K. V., Santos, E. M., Bussadori, S. K \& Martin, M. D. (2007). Avaliação radiográfica da qualidade de tratamentos endodônticos realizados por especialistas de um plano de saúde odontológico. Conscientiae Saúde. 6(2): 371-375.

Gonçalvez, I. Q. A. (2007). Avaliação dos tratamentos endodônticos realizados no curso de especialização da faculdade de odontologia de Piracicaba Unicamp de 1997 a 2001. Piracicaba, 73f. Dissertação (Doutorado) -Universidade Estadual de Campinas.

Grecca, F. A. (2008). Avaliação da qualidade das obturações endodônticas realizadas por estudantes de graduação. Rev. Fac. Odontol. 49(3): 24-27.

Imura, N. (2004). Fatores de sucesso em endodontia: análise retrospectiva de 2.000 casos clínicos. Revista Associação Paulista de cirurgiões Dentistas. 58 (1):29-34.

Lage-Marques, J. L., Fenyo-Pereira, M \& Sanfioti, L. M. L. (1996). Análise radiográfica da qualidade do tratamento endodôntico e suas interações. Revista Brasileira de Odontologia. 53 (3):11-15.

Lolpes, H. P \& Squeira-Júnior, J. F. Endodontia: biologia e técnica, 2.ed. Rio de Janeiro: Guanabara Koogan, 2004.

Louzada, L. M., Arruda-Vasconcelos, R., Duque, T. M., Casarin, R. C. V., Feres, M. \& Gomes, B. P. F. A. (2020). Clinical Investigation of Microbial Profile and Levels of Endotoxins and Lipoteichoic Acid at Different Phases of the Endodontic Treatment in Teeth with Vital Pulp and Associated Periodontal Disease. J Endod. 46 (6):736-747.

Menezes, M. R. A., Alves-Silva, E. G., Santana, E. C. G \& Mendes, V. C. (2021). Randomized clinical studies of the benefits and limitations of Irradiation Laser Intravenous of Blood (ILIB) in Dentistry: an integrative review. Research, Society and Development. 10 (2): e30910212576.

Neves, B. R., Chaves, A. T. D., Veloso H. H. P., Alves-Silva, E. G \& Dourado, A. C. A. G. (2020). Evaluation of the prescription of antibiotics by endodontists in acute periapical abscess. Research, Society and Development. 9 (9): e826997901.

Patriota, E. C. R., Amorim, V. S. C. M., Arruda-Vasconcelos, R. Louzada, L. M., Menezes, M. R. A., Gomes, B. P. F. A \& Alves-Silva, E. G. (2020). Efficacy of guided endodontics in treating teeth with radicular calcification: integrative review. Research, Society and Development. 9 (8): e655986066.

Pereira, C. V \& Carvalho, J. C (2008). Prevalência e eficácia dos tratamentos endodônticos realizados no Centro Universitário de Lavras, MG -uma análise etiológica e radiográfica. $R F O .13$ (3): 36-41.

Souza, R. A. (2006). Análise crítica do papel da obturação no tratamento endodôntico. Jornal Brasileiro de Endodontia. 6 (23): 29-39.

Travassos, R. M. C., Albuquerque, D. S., Caldas-Júnior, A. F \& Santos, R. (2005). Avaliação da terapia endodôntica. Odontologia. Clín-Científ. 4 (3):189-192. 